\title{
Prevalance of Musculoskeletal Pain Among Judo Players
}

\author{
Zaki Tayebali', Dr. Preeti Lendghar ${ }^{2}$, Dr. Tushar J Palekar ${ }^{3}$ \\ ${ }^{1}$ Research Scholar, Dr D Y Patil College of Physiotherapy, Pune, India \\ ${ }^{2}$ Assistant Professor, Dr D Y Patil College of Physiotherapy, Pune, India \\ ${ }^{3}$ Professor and Principal, Dr D Y Patil College of Physiotherapy, Pune, India
}

\begin{abstract}
Background: injuries ${ }^{2}$.

\section{Objectives:}

1) To identify various sites of pain with the help of a questionnaire

2)To find the intensity of pain using NPRS

3) To find if the pain causes any activity limitations.
\end{abstract}

Practice of judo integrates varied situations of physical contact and specific requirements, which makes it a competitive modality with a high rate of musculoskeletal injuries. When combined with continuous requirement for physical and technical improvement that are common in competitive physical training, these demands can constitute physical training, and furthermore can predispose the individuals to musculoskeletal

Judo was appointed as a sport that has a prominent relative risk of injury compared to other sports.

\section{Materials and Methodology}

A questionnaire based study comprising of 50 judo atheletes. The subjects were each given a questionnaire. The purpose of the study was explained to all the participants and an informed consent was taken from each subject. Outcome measure i.e questionnaire which was ethically approved was explained to all the participants in the language comfortable to them and they were asked to fill the questionnaire.

Results:

Shoulder was the most common site of pain as recorded from this study. The second most common pain being lower back pain. Followed by knee, hip \& foot and ankle in that order. The least common being upper back.

\section{Conclusion:}

The study concludes that most of the judo athletes complained of shoulder pain. The throwing technique was the most common maneuver causing the pain.

Keywords: Judo players, Musculoskeletal Pain, Judo, Questionnaire

\section{INTRODUCTION}

One of the major negative aspects of competitive sports is excessive strain on the body which often leads to bodily injuries. Over the last years the incidence of sports injuries has increased due to growing physical proficiency.

A majority of people experience back pain at some point in their lives, and a demanding contact sport like judo can end up leading to musculoskeletal pain 
through accidents, overwork or errors in the technique. ${ }^{1}$

Practice of judo integrates varied situations of physical contact and specific requirements, which makes it a competitive modality with a high rate of musculoskeletal injuries. When combined with continuous requirement for physical and technical improvement that are common in competitive physical training, these demands can constitute physical training, and furthermore can predispose the individuals to musculoskeletal injuries².

Judo was appointed as a sport that has a prominent relative risk of injury compared to other sports.

Judo is a contact sport, therefore, injuries are fairly common. Some of the most common judo injuries are:

- Back Pain

- Slipped disk

- Spinal injuries

- Knee injuries-Ligament damage, ligament sprains, ligament tears, cartilage tears.

- Dislocations

- Shoulder injuries- AC joint sprain, dislocation, impingement syndrome.

Judo manoeuvres frequently include lifts, strikes and throws. These are used both in competition and during sparring, so the possibility of injury can be high. The focus on landing on the back plus constant twisting and stretching can be damaging, especially if the muscles are not up to the task. Previous back pain can be exacerbated by judo.

Falling techniques have a major impact. Correct falling is essential for maintaining safe weight distribution throughout the body when landing from throws. By practising in a correct and appropriate manner, the weight can be distributed to areas like the arms and legs, further reducing the probability of musculoskeletal injuries and pain. ${ }^{3}$

A related cause is the technique of the thrower. Inadequate throwing or lifting can cause back pain to either the thrower or the faller. For example the faller may land awkwardly due to a sloppy throw, or the thrower might make the lift with their back rather than their legs. Lifting should always come from the legs and not the back, with the athletes close together.

Pain is a sensation of subjective and individual nature and can assume different forms and intensity. Excessive stress placed upon the body during trainings can lead to bodily injuries. Many injuries can be prevented by practising correct joint stability, controlling muscular strength and increasing flexibility. Stretching exercises should be an integral part of training.

Majority of the athletes experience chronic pain in the musculoskeletal system regardless of their age, proficiency or years of training. It is proof that overtraining is very common in judo competitiors. ${ }^{4}$

Low back pain is a common musculoskeletal disorder affecting the general population as well as athletes. Low back pain is divided into specific and non specific low back pain. Specific low back pain is relatively uncommon and it is caused by malignancies, infections, inflammatory, spondyloarthropathies and fractures. Conversely non specific low back pain, back pain that can be attributed to a specific cause, is quite common. With previous studies indicating a prevalence in the ranges of $40 \%$ to $60 \%$ for the general population and $30 \%$ to $85 \%$ for the athletes. Although the prevalence of non specific low back pain varies depending on the sport, it is commonly observed in all sports including judo ${ }^{5}$ 


\section{METHODS AND MATERIAL}

Study Location: Study was conducted among Judo players in Mumbai and Pune, India.

Sampling Method: 50 Judo atheletes were selected based on the years of practice and withing the age group of 15-30.

Procedure and data collection: 50 judo athletes of age group 15-30 years were selected in the study, fulfilling the inclusion criteria. The purpose of the study was explained to all the participants and an informed consent was taken from each subject. Outcome measure i.e questionnaire which was ethically approved was explained to all the participants in the language comfortable to them and they were asked to fill the questionnaire.

\section{RESULTS AND DISCUSSION}

In this study, 50 judo athletes fulfilling the criteria were given a questionnaire and the results were graphically represented.

\section{Graph No. 1}

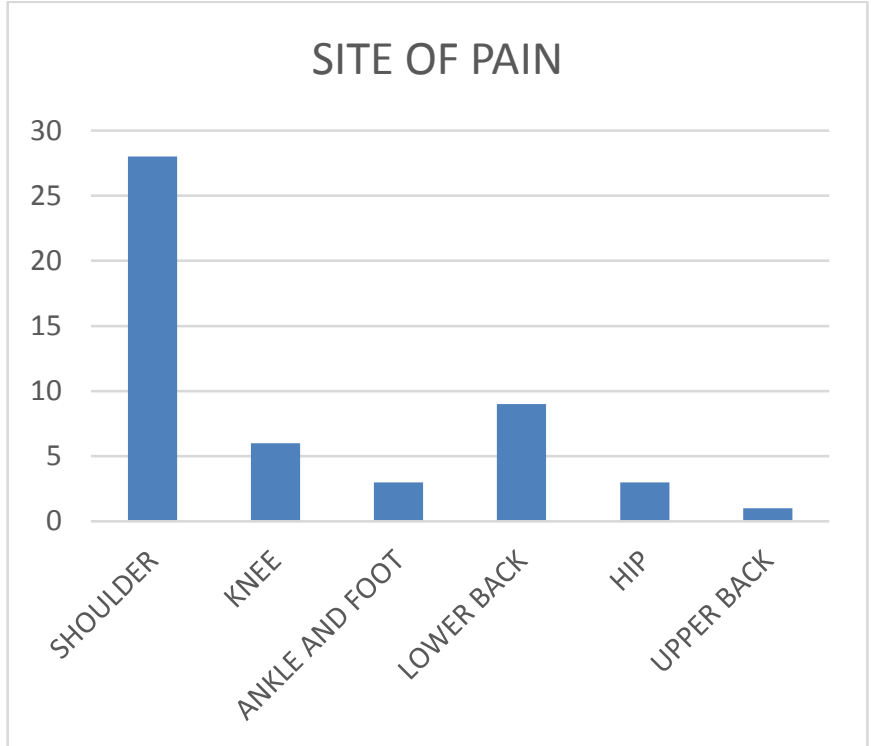

Interpretation: The above graph no.1 shows the most common site of pain experienced. According to this study, shoulder pain is seen to be the most common.

\section{GRAPH NO.2}

\section{MANUEVER CAUSING THE PAIN}

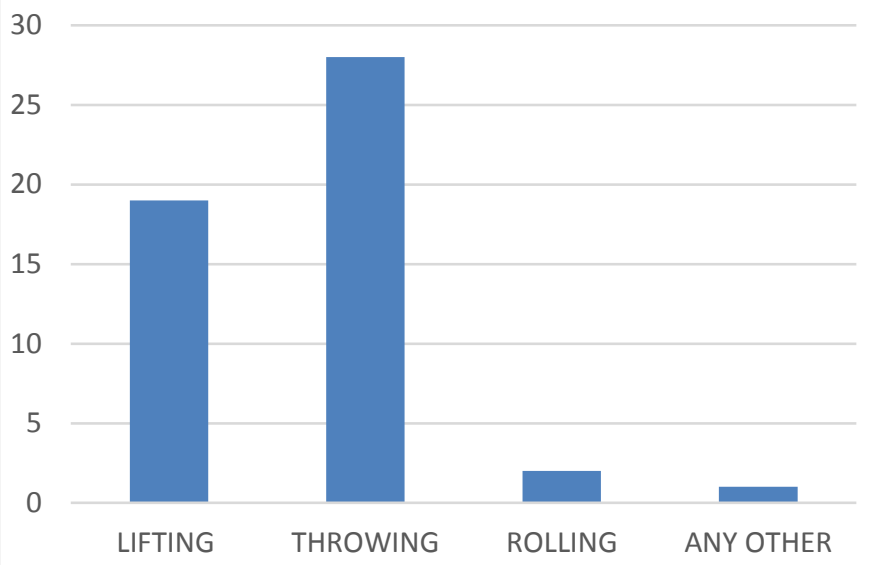

Interpretation: The above graph no. 2 shows the most common maneuver causing the pain. According to this study, Throwing was the most common maneuver

\section{GRAPH NO.3:}

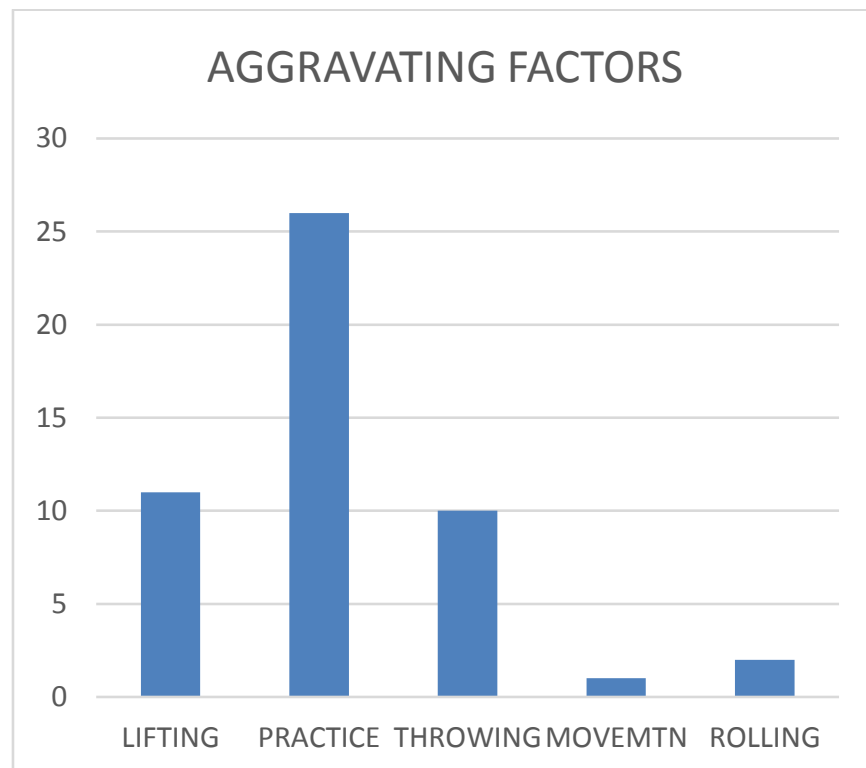

Interpretation: The above graph No.3 shows the most common aggravating factors for the pain and this 
study concludes practicing judo to be the most common factor.

\section{GRAPH NO.4:}

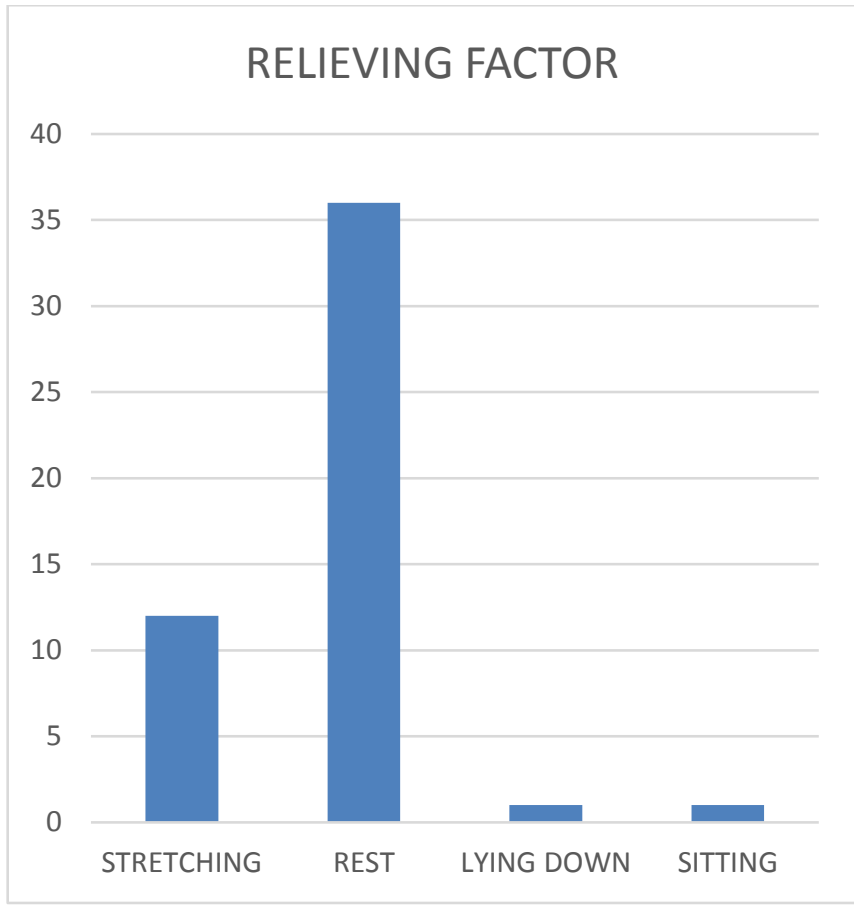

Interpretation: The above graph No.4 shows the most common relieving factors for the pain and this study concludes rest to be the most common.

\section{GRAPH NO.5:}

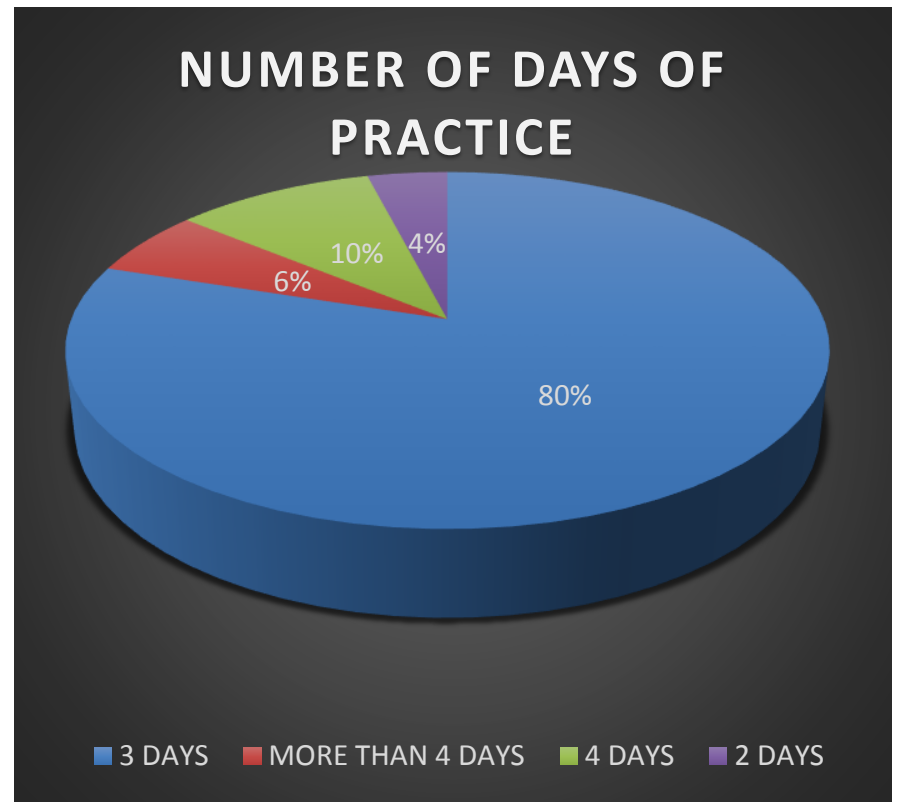

Interpretation: The above graph no. 5 shows the number of days of practice in a week. Here, the most is 3 days a week.

\section{GRAPH NO.6:}

\section{NPRS \\ AT REST VS ON ACTIVITY}

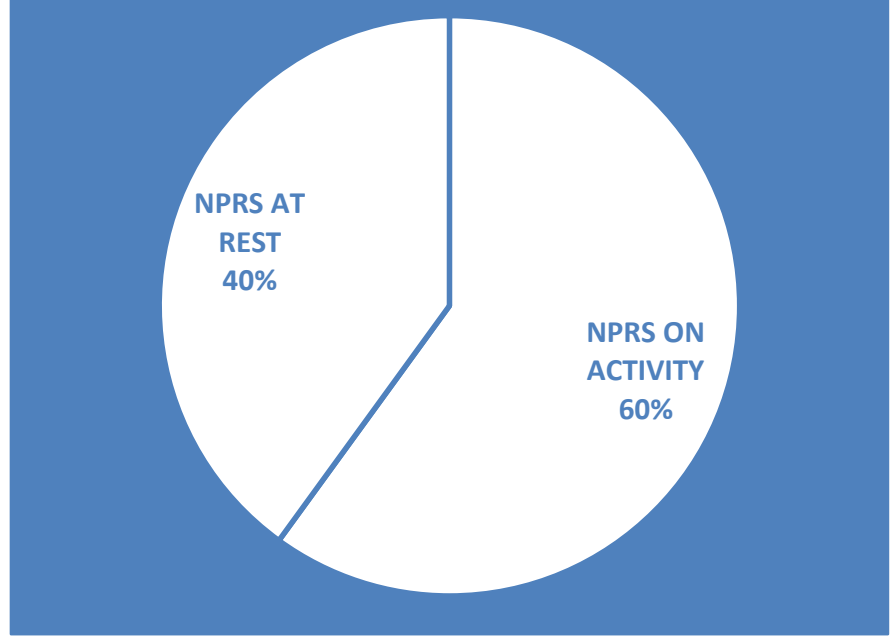

Interpretation: The above graph no.6 shows the average NPRS on activity and at rest. The pain is seen more on activity and lesser at rest.

\section{GRAPH NO.7:}

\section{MALE : FEMALE RATIO}

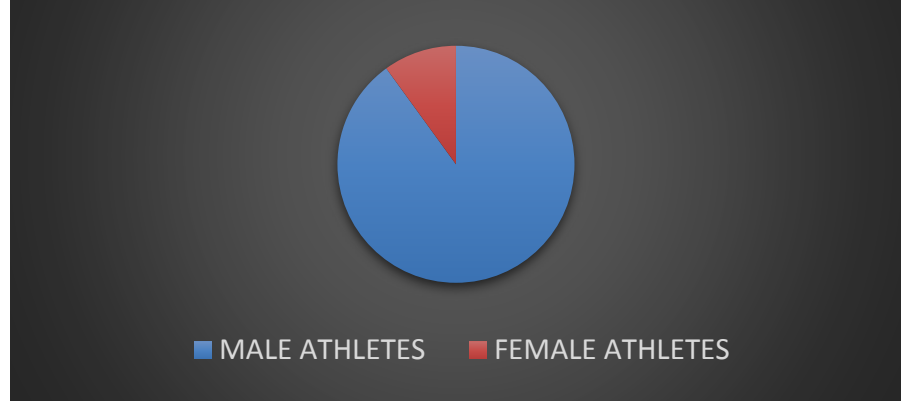

Interpretation: The above graph no. 7 shows the male : female ratio. Wherein the male athletes were more than the female athletes in this project. 


\section{DISCUSSION}

The present study on "Prevalance of Musculoskeletal Pain among Judo players" was conducted to determine which was the most common site of pain experienced by judo players. The study was conducted with 50 judo athletes.

The objective of this study was to determine the most common site of pain, to find the intensity of pain and if the pain caused any activity limitations.

According to our study, the most common musculoskeletal pain seen among judo players was shoulder pain. The maneuver that was the most common was the throwing maneuver.

Recent studies have shown that, shoulder injuries can result from a strong impact after a knock during a fall or of a fall on his opponents, causing overload once the impact involves your weight with added strength and speed. The fingers are subjected to differentiated aggression in the moment when there is a contest for the best grip, allowing athletes to apply the most appropriate technique, or simply to be more aggressive in combat ${ }^{4}$.

The most common aggravating factor for pain seen in these athletes was while practicing judo. According to recent studies, for improvement of athletic performance, training overloads must come along with a minor fatigue and acute reductions in performance, alternating with appropriate recovering periods: When excessive training overload is combined with inadequate recovery, the overtraining state happens ${ }^{5}$.

According to NPRS, the athletes in this study complained of considerable increase in pain $(60 \%)$ during activity and lesser pain (40\%) at rest.
The most on an average the athletes practiced judo thrice a week.

According to a recent study, it is suggested that an athlete should not practice for more than 7 hours a week in order in minimise the occurrence of musculoskeletal injuries.

Since the greater number of injuries occurred during the training of athletes, it is suggested that athletes include in their training sessions an appropriate period of warm-up and return to rest, also including proprioceptive and plyometric exercises that simulate the most the movements common to these modalities as a way of preventing injuries and improving physical performance of the athletes ${ }^{5}$.

\section{IV.CONCLUSION}

The study concludes that that occurrence of shoulder pain was greater among the athletes with lower back being the 2nd most common.

It also concludes that pain was more during judo practice.

\section{REFERENCES}

[1]. Minghelli B, Isidoro R Prevalance of Injuries in Jio-Jitsu and Judo Athletes Of Portugal South: Associated Injury Mechanisms, Journal of Community Medicine and Health Education, June 15,2016

[2]. Ana Laura Gil Manzato, Hugo Parra de Camargo et al Musculoskeletal Injuries in Judo Practitioners, Fisioter Pesqui, 2017;24(2):127134

[3]. Elena Pocecco, Gerhard Ruedl, Nemanja Stankovik, Stainslaw Sterkowicz et al Injuries in Judo: a systemic literature review including suggestions of prevention, British Journal og Sports Medicine 2013;47:1139-1143 
[4]. Dariusz Boguszewski, Malgorzata Buda et al Chronic Pain in the Musculoskeletal system among Judo athletes, Journal of Combat Sports and Martial Arts 2014;2(2); Vol.5 77-82

[5]. Okada.T, Nakazato.K et al Body mass, non specific low back pain, and anatomical changes in the lumbar spine in judo athletes. 2007;37(11):688-93

\section{Cite this article as :}

Zaki Teyebali, Dr. Preeti Lendghar, Dr. Tushar J Palekar, "Prevalance of Musculoskeletal Pain Among Judo Players", International Journal of Scientific Research in Science and Technology (IJSRST), Online ISSN : 2395-602X, Print ISSN : 2395-6011, Volume 6 Issue 4, pp. 07-12, July-August 2019. Available at doi : https://doi.org/10.32628/IJSRST196350 Journal URL : http://ijsrst.com/IJSRST196350 